Relations industrielles

Industrial Relations

\title{
Making Management Human. By A.J. Marrow. New York:
} McGraw-Hill, 1957, 241 pp.

\section{Rodrigue Giroux}

Volume 13, numéro 2, avril 1958

URI : https://id.erudit.org/iderudit/1024002ar

DOI : https://doi.org/10.7202/1024002ar

Aller au sommaire du numéro

Éditeur(s)

Département des relations industrielles de l’Université Laval

ISSN

0034-379X (imprimé)

1703-8138 (numérique)

Découvrir la revue

Citer ce compte rendu

Giroux, R. (1958). Compte rendu de [Making Management Human. By A.J.

Marrow. New York: McGraw-Hill, 1957, 241 pp.] Relations industrielles /

Industrial Relations, 13(2), 238-239. https://doi.org/10.7202/1024002ar

Tous droits réservés (C Département des relations industrielles de l’Université Laval, 1958
Ce document est protégé par la loi sur le droit d'auteur. L’utilisation des services d'Érudit (y compris la reproduction) est assujettie à sa politique d'utilisation que vous pouvez consulter en ligne.

https://apropos.erudit.org/fr/usagers/politique-dutilisation/ 
Faut-il ajouter que les accidents de travail, de la rue et les infirmités congénitales fournissent annuellement, en moyenne, 250,000 handicapés de plus.

On est parfois enclin à croire que ce sont les guerres qui fournissent le contingent le plus considérable de personnes handicapées. Durant la dernière guerre, il y eut 20,500 amputés chez les militaires; durant cette période, les civils en comptaient 120,$000 ; 1,500$ des forces armées devinrent aveugles et pour les civils, 60,000 .

D'autres exemples sont donnés montrant l'évidence de ce problème. Les auteurs font une brève description du programme fédéral de réhabilitation, de celui des anciens combattants et des organismes des Etats américains sur qui repose, en définitive, la réalisation pratique de la réhabilitation.

Les auteurs, en terminant cette partie, soulignent l'aspect économique de la réhabilitation. Il est admis que pour chaque dollar dépensé par le Gouvernement fédéral à la réhabilitation, le travailleur handicapé remis dans le circuit économique paiera $\$ 10.00$, en taxes, au Gouvernement fédéral.

Ce volume contient de plus trois appendices dans lesquels les auteurs décrivent le système de compensation en Angleterre et en Ontario. Dans quelques lignes, ils résument trop brièvement le service de réhabilitation de la Commission des Accidents du Travail de l'Ontario, situé à Malton, lequel est, selon nos vues, le mieux organisé au Canada.

Les auteurs sont des économistes, et versés dans l'administration des assurances sociales. Dans ce volume, ils s'attardent à traiter de question d'assurabilité, de paiement de prestations aux accidentés, enfin à tout ce que comporte l'assurance-accidents.

Sans doute est-il question de réhabilitation. En fait, les auteurs consacrent une cinquantaine de pages à ce sujet; ils résument ce qui s'est fait aux EtatsUnis.

Nous croyons que ce volume peut intéresser plus particulièrement les spécialistes adonnés aux questions d'assuranice-accidents.

\section{C.-RAymond Maranda}

Making Management Human. By A.J. Marrow. New York: McGraw-Hill, 1957,241 pp.

M. Marrow est un psychologue, professeur au «New School for Social Research », et qui par surcroît est président de la «Harwood Manufacturing Corporation». Dans son volume, il rapporte les résultats qui furent atteints dans sa corporation depuis environ vingt ans, grâce à l'application de principes psychologigues aux différentes phases de l'administration du personmel. Marrow comprend parmi ses collaborateurs un chercheur comme Alex Bavelas. Dans de telles circonstances, les recherches entreprises sont toujours intéressantes, de même que les résultats.

Tout au long de son exposé, Marrow n'hésite pas à blâmer la gérance de l'entreprise si elle n'obtient pas de ses employés la coopération à laquelle elle s'attend. "Too few executives in industry today have an adequate conception of the values the average man seeks in work, or the different meanings work has for different people... The success of today's executive depends on cooperation in his organization, or his skill in getting things done by, for and through people ». Comme de nombreux exécutifs ignorent les besoins psychologiques de leurs subalternes, ils obtiennent le conformisme mais non la coopération, car ils ne saisissent pas le sens du mot motivation. Par suite de l'application grandissante des principes de l'automation, des situations déjà tendues vont certainement éclater. En effet, l'employé, délivré de ses préoccupations techniques, verra de plus en plus ses pensées accaparées par les problèmes psychologiques de sa position.

Par suite de leur comportement, certains patrons ne savent pas ce qui se passe réellement. Ordinairement, l'entourage rapporte au patron les informations qu'il s'attend à recevoir. Par son comportement agressif, autoritaire, et même antagoniste, il n'a jamais appris à écouter et encore moins à comprendre. Seul il connaît la solution. Il commencera à parler avant que son interlocuteur ait terminé son propre exposé. Dans les conférenoes ou les autres réunions. il prend la parole, il la conserve. Il exige et obtient un consentement unanime guand il propose un changement quelconque. Comme l'ont fait si bien 
remarquer Carl. R. Rogers et F. J. Roethlisberger, le patron, le meneur, ne conçoit pas qu'il arrête le flot des communications par sa tendance compulsive à évaluer, à approuver ou désapprouver les autres sans les écouter ni les comprendre.

Marrow consacre plusieurs chapitres à expliquer cette attitude négative du «leader». Il l'illustre avec plusieurs exemples concrets. Mais comment changer l'attitude du 《leader»; comment surtout l'amener à comprendre ses pro. pres faiblesses? Marrow propose la méthode des conférences. Chaque conférence est analysée avec les techniques suivantes:

a) la formule de réaction: «PostMeeting Reaction Form »;

b) l'indice de participation;

c) l'observateur indépendant;

d) l'enregistrement sur bande sonore;

e) la discussion circulaire.

Marrow parle beaucoup de participation. Il montre comment de nombreux problèmes furent solutionnés à Harwood en obtenant la participation des employés. Il en loue les effets constamment, mais il ignore un fait important. Si la participation a pour but véritable de manipuler les employés afin de leur faire accepter des transformations auxquelles ils s'opposent par principe, à ce moment l'échec est inévitable. Les malaises qui tourmentent l'organisation ne peuvent pas être éliminés s'ils résultent de l'incompétence de la gérance.

La thèse de Marrow est vivante; elle essaie de solutionner un problème aigu. Toutefois, elle y aurait beaucoup gargné si elle avait été exposée de façon plus systématique et plus vigoureuse. Il aurait été préférable d'énoncer d'abord les principes psychologiques et de les expliquer ensuite par des exemples. L'auteur a probablement voulu vulgariser la science psychologique afin d'en rendre les principes plus acceptables pour les patrons et les meneurs de l'industrie. L'effet a peut-être été manqué, car l'exposé de sa doctrine est trop souvent interrompu par des études de cas. Ceux-ci, quoique bien choisis, sont évidemment trop nombreux. Il en est de même de tout bon vin: quand on en boit trop, il perd de sa saveur.

Rodrigue Giroux
The Big Business Executive. By Mabel Newcomer. New York: Columbia University, 1955. 164 pp. \$4.25.

Cet entrefilet a paru dans la page financière d'un journal quotidien bien connu: «M. Joseph Dupont a été élu à la présidence de la Compagnie Durand \& Durand, Ltée. Il assumera également les fonctions de président du conseil d'administration ». Plusieurs lecteurs se demanderont comment $\mathbf{M}$. Dupont est parvenu à obtenir une telle position. Le livre de Newcomer fut spécialement conçu pour répondre à cette question.

Jusqu'à date, la plupart des études sur la position de président et de viceprésident ont surtout porté sur la nature de la position, c'est-à-dire sur les fonctions impliquées. Seulement quelquesunes ont scruté les origines sociales de nos meneurs d'entreprises depuis 1900 . Quelle était leur expérienoe globale, leur entraînement? Quel niveau d'éducation ont-ils atteint? Leur entrainement et leur éducation répondaient-elles aux exigenoes de la position? Les qualifications requises variaient-elles suivant le genre d'entreprise? Quels sentiers ces nouveaux présidents ont-ils suivis pour atteindre leur but? $\mathrm{Y}$ sont-ils parvenus parce qu'ils étaient les candidats les mieux qualifiés, ou par suite de leur ancienneté et de leur influence familiale? Pour répondre à ces questions, l'auteur a choisi une coupe longitudinale des présidents des grandes entreprises américaines depuis 1898 jusqu'à 1953. Cette coupe, il l'analyse au moyen de trois échantillons couvrant les années $1900,1925,1950$. Ces échantillons in-. cluent les présidents et les vice-présidents des conseils d'administration des. compagnies dont l'actif était de plus de $\$ 75,000,000$ en $1950, \$ 50,000,000$ en 1925 et $\$ 25,000,000$ en 1900 .

L'analyse qui en résulte nous livre des faits intéressants que l'auteur n'ose pas trop commenter. En effet, il se défend bien de vouloir le faire: Le document est court et facile à lire. Il laisse entrevoir à quelle politique sociale l'ont peut s'attendre de l'exécutif de la plupart des compagnies quand leurs présidents répondent à un stéréotype bien défini. Le portrait suivant, quoique basé sur des données moyennes, semble bien définir le président typique bien connu. 